\title{
Burnout and Depressive Symptomatology of the Employees in Institutions of Chronic Diseases
}

\author{
Dr. Vasiliki Brouskeli \\ Assistant Professor in Health Psychology, \\ Democritus University of Thrace, Greece \\ Eustathios Giakovis \\ Msc in Counselling in Special-General Education and Health, \\ University of Thessaly, Greece \\ Prof. Maria Loumakou \\ Professor in Health Psychology, \\ National and Kapodistrian University of Athens, Greece
}

Doi: $10.1515 / \mathrm{mjss}-2017-0038$

\begin{abstract}
The purpose of the research is twofold: a) to determine whether the employees in Greek Institutions of Chronic Diseases show burnout and depressive symptomatology and to connect them with sociodemographic characteristics and b)to investigate the relation between these two health factors. The participants completed a Greek version of Maslach's Burnout Inventory (MBI), a Greek version of Beck's Depression Inventory (BDI), and a questionnaire regarding the socio-demographic characteristics. According to the results of the research, a significant percentage of participants show burnout and depressive symptomatology. The exhaustion was found to be associated with gender, age, employment and marital status. The scientific staff has less depressive symptoms than the auxiliaries. Finally, higher levels of burnout effect on higher levels of depressive symptomatology. Based on the results, it is necessary to make interventions on a preventive level as well as to deal with burnout problems and depression of employees on time. In conclusion, there is a need to conduct further researches that, among others, check the targeting and the effectiveness of interventions.
\end{abstract}

Keywords: Institutions, Chronic Diseases, depression, burnout

\section{Introduction}

The Institutions of Chronic Diseases in Greece constitute health and care providers. Their purpose is the nursing and the functional, social, pre-vocational and vocational rehabilitation of persons with intellectual disability or chronic mobility impaired with intellectual disability, and therefore suffering from a total or partial incapacity of performing on their own the tasks of everyday life. In these institutions are working, among others, Administrative Staff, Doctors, Psychologists, Nurses, Physiotherapists, Health Visitors, Social Workers, Occupational Therapists, Speech Therapists, Dietitians, Cooks, Drivers, Guards and patient transport staff (Official Gazette of the Hellenic Republic 2009). 


\subsection{Burnout and healthcare providers}

"Burnout is a psychological syndrome of emotional exhaustion, depersonalization, and reduced personal accomplishment that can occur among individuals who work with other people in some capacity" (Maslach et al. 1997, p.191). Increased feelings of emotional exhaustion, depersonalization (i.e., negative, cynical attitudes and sentiments about one's clients) and the tendency to evaluate oneself negatively, especially regarding one's work with customers are the three fundamental aspects of the syndrome. The potential consequences of burnout may be severe for workers, their clients and the institutions they work for (Maslach et al. 1997).

Burnout has been widely studied into service providing professions, and specifically at healthcare facilities. Its consequences are detrimental, not only to the healthcare professional at a personal level but also to the healthcare service organisation, and of course to the degree of the provided health services to the people (Pappa et al. 2007). It has been widely studied amongst the healthcare staff, and the following seem to be predictors of burnout among nurses: gender, age, years of experience at work, working in more than one institution, being involved in management positions, job satisfaction, hardiness, and experience of work-home and home-work interaction (Queiros et al. 2013).

\subsection{Depression and HHealthCare Providers}

Depression is a frequent and severe medical illness that negatively affects people's lives, causing feelings of sadness and a loss of interest in activities once enjoyed. It may lead to emotional and physical problems as well as to decreased abilities to function at work and home (Parekh 2017).

Depression, as an issue requires researching attention and appropriate intervention; it has been studied in several health care professionals. For instance, it has been investigated in nurses (Chang et al. 2010), nursing technicians and nursing assistants (Rios et al. 2010), physicians (Levine and Bryant 2000), social workers (Siebert 2004), and intellectual disability support staff (Mutkins et al. 2011). In the literature review of Koinis and his colleagues (2014), it is being accented that the working conditions of the health professionals, working hours, disturbed or minimum communication with co-workers and lack of sleep can contribute to the development of psychiatric symptoms and long-term to depression. In the current bibliography, the rates of depression symptoms in health care professionals differ importantly, depending on the methodology followed every time as well as the country where the research is conducted (for instance: Abbas et al. 2013; Gao et al. 2011; Chang et al. 2010).

\subsection{Burnout and depression}

Burnout and depression are separate entities, although they may share several "qualitative" characteristics. Burnout may lead to a general negative attitude towards life and, in this sense, it shares common features with depression (lakovides et al. 2003).

Burnout is more than depression, a multidimensional phenomenon, but, unlike depression, restricted to the job setting (Schaufeli and Buunk 2003), whereas depression is a context-free clinical syndrome and characterised by more global attributions (Bakker et al. 2000; Leiter and Durup 1994). Furthermore, a reduced sense of superiority and a perceived loss of status are more characteristic for depressed than for those who are burnt-out (Brenninkmeyer et al. 2001). The relationship between burnout and depression has been studied in numerous of studies and an association between the two of them has been observed (Ahola et al. 2014; Fong et al. 2016; Toker and Biron 2012). Additionally, in some studies, the distinctiveness between these two terms is not always attainable. (Bianchi et al. 2015; Schonfeld and Bianchi 2016).

\section{Purpose of the Study}

To our knowledge, the researches in Greece concerning burnout and depressive symptomatology, conducted within private and public hospitals, whilom about the first issue (Alexias et al. 2010; 
Noula et al. 2010; Tragea et al. 2012) and whilom about the second issue (Kaklamanou et al. 2014; Tselebis et al. 2006; Kyloudis et al. 2010). We are not aware of research that has been conducted related to these two issues with the same sample. Moreover, we are not conscious of research relevant to burnout and depressive symptoms within an Institution of Chronic Diseases in Greece. The purpose of the particular study is to investigate the burnout and depressive symptomatology of the employees within Greek Institutions of Chronic Diseases concerning their sociodemographic and work-related characteristics and to examine the relation between these two health factors as well.

\section{Methodology}

\subsection{Participants}

A total of 100 employees (30\% male and 70\% female) from four Institutions of Chronic Diseases in semi-rural areas (Karpenisi, Larissa, Trikala and Fthiotida) took part in the research. Thirty percent of the one hundred participants in the research were male and $70 \%$ female. A percentage of $10 \%$ of them was between the age 18-30, 39\% between $31-42$ years old, $45 \%$ between $43-54$ years old, and $6 \%$ was 55 years or older. As for the working position, $15 \%$ of them had administrative duties, $22 \%$ were specialised scientific staff, $27 \%$ nurses, and $36 \%$ auxiliary staff. It is being clarified that the "special scientific staff" refers to specialities that provide care services to patients, such as Psychologists, Social Workers, Physiotherapists and Occupational Therapists. The nursing staff was a particular category of scientific staff in the study, as it is a professional team with a special research interest, which has been widely investigated and found to be affected by burnout and depression in various ways. Regarding the level of education, $12 \%$ of the participants were primary school graduates, $39 \%$ were high school graduates, $41 \%$ graduates of Universities or Technological Educational Institutes, and $8 \%$ of them had a Master's or Doctorate diploma. A percentage of $19 \%$ of the participants was working within the Institutions for Chronic Diseases up to three years, $26 \%$ from 4 to 9 years, and $55 \%$ from 10 years and above. Regarding the marital status of the participants, $14 \%$ of them were unmarried, $75 \%$ married and $11 \%$ divorced. Finally, $20 \%$ of participants had no children, $21 \%$ had one child, $33 \%$ had two children and $26 \%$ three or more children.

\subsection{Measures}

The Maslach Burnout Inventory (MBI) of Maslach and Jackson (1981) and in particular a customised version in Greek (Anagnostopoulos and Papadatou, 1992), was used for the measurement of burnout. The tool includes 22 issues/recommendations that measure three original dimensions of burnout: the emotional exhaustion, the depersonalisation, and the reduced personal accomplishment. The answers are given by a Likert-type scale with seven graduations and we used the suggested subscales categorisation of Anagnostopoulos and Papadatou (1992), dividing in groups of small, medium or high burnout levels .

The reliability of internal consistency (index Cronbach's a) for the emotional exhaustion subscale is .84 , for the depersonalization subscale is .55 , and for the reduced personal accomplishment subscale is .71 (Papadatou et al. 2012, pp.220-221). Minimum phrasal modifications were made to the tool, based on the use of the tool from another Greek study (Tsiros 2009), mainly because of the particular sample's needs (e.g. the term "cared" was used instead of "patient"). In this particular study, Cronbach's a was equal to .92 for the emotional exhaustion subscale, equal to .79 for the depersonalization subscale and equal to .70 for the reduced personal accomplishment subscale and is considered satisfactory for each case.

To measure the depressive symptomatology, Beck's Depression Inventory (Beck Depression Inventory, BDI) was used, a self-completion scale for the estimation of the depressive symptomatology's severity, consisting of twenty-one subjects (Beck et al. 1961). The Greek version of the scale has been standardised for adults. It consists of 21 ascertainments that describe symptoms such as pessimism, sense of failure and loss of satisfaction. The data, in this case, also 
showed the satisfactory reliability of internal consistency as Cronbach's a coefficient is equal to .90. For the Greek version of the scale, the threshold value for the detection of clinical cases is the value 21, while the values 21-30 indicate slight depression and values between 31-40 indicate moderate depression (Tzemos 1984). Finally, for the socio-demographic characteristics of the participants (and in particular gender, age, job, education, years of professional employment in the Institutions of Chronic Diseases, marital status and number of children) a questionnaire was created.

\subsection{Procedure}

After communication with the Management of the Institutions, the license for the dispensing of the questionnaire to the staff was given. Furthermore, the participants were informed by letter preceding the survey, that there are no right or wrong answers. It was clarified in writing that the completion of the questionnaire was voluntary and it lasted on average twenty minutes. Finally, the participants were informed about the ways of being informed about the results of the research.

Besides the descriptive statistical analysis, an inductive analysis of data was made, and in particular T-test analysis for independent samples and analysis of variance ANOVA (One-way ANOVA), to make the comparisons between the different groups, as those derived from the values of the demographic variable. Pearson's $x 2$ test to investigate the existence of dependence or not between burnout and demographics and Pearson's $r$ correlation coefficient to test the presence of the linear relationship between Burnout determinants and Depressive Symptomatology scores. Before carrying out these specific statistical analyses, the conditions of their implement were checked.

\section{Results}

\subsection{Dimensions of Burnout and Depressive Symptomatology}

Descriptive results in Table 1 present the levels of experienced burnout levels of the participants.

Table 1. MBI factors descriptive results

Experienced Burnout

\begin{tabular}{lcccccccc} 
& Low & $\mathrm{N}(\%)$ & Medium & $\mathrm{N}(\%)$ & High & $\mathrm{N}(\%)$ & $\mathrm{M}$ & $\mathrm{SD}$ \\
\hline Emotional exhaustion & $\leq 20$ & $29(29.0 \%)$ & $21-30$ & $27(27.0 \%)$ & $\geq 31$ & $44(44.0 \%)$ & 30.08 & 13.51 \\
Depersonalization & $\leq 5$ & $21(21.0 \%)$ & $6-10$ & $39(39.0 \%)$ & $\geq 11$ & $40(40.0 \%)$ & 10.55 & 5.71 \\
Personal accomplishment & $\geq 42$ & $60(60.0 \%)$ & $41-36$ & $15(15.0 \%)$ & $\leq 35$ & $25(25.0 \%)$ & 42.24 & 9.74 \\
\hline
\end{tabular}

Based on the above, by calculating the percentage of individual experiencing high levels of emotional exhaustion and depersonalization and low levels of personal accomplishment's feeling it appears that $13 \%$ of the employees $(\mathrm{N}=13)$ suffer from burnout syndrome. The data of Table 2 show the classification of the participants' depressive symptomatology.

Table 2. Depressive symptomatology descriptive results

\begin{tabular}{llcccc} 
& & Count & $\mathrm{N}(\%)$ & $\mathrm{M}$ & $\mathrm{SD}$ \\
\hline & Absence of D.S. & $\leq 5$ & $33(33.0 \%)$ & & \\
Vepressive Symptomatology & $6-20$ & $55(55.0 \%)$ & & \\
& Very light & $21-30$ & $9(9.0 \%)$ & 10.12 & 8.78 \\
& Light & $31-40$ & $1(1.0 \%)$ & & \\
& Moderate & $\geq 40$ & $2(2.0 \%)$ & & \\
\hline
\end{tabular}

\subsection{The Effect of Demographic Factors on Burnout}

Table 3 summarizes the average scores (and standard deviations) of each factor of Burnout 
according to the demographic characteristics of the participants, as well as the inductive analyses results on the significance of their effect.

Table 3. Demographics effect on the factors of Burnout

\begin{tabular}{|c|c|c|c|c|c|c|c|}
\hline \multirow{4}{*}{ Sex } & \multirow{4}{*}{$\begin{array}{l}\text { Male } \\
\text { Female }\end{array}$} & \multicolumn{2}{|c|}{ Emotional Exhaustion } & \multicolumn{2}{|c|}{ Depersonalization } & \multicolumn{2}{|c|}{ Personal accomplishment } \\
\hline & & $\mathrm{M}$ & SD & $M$ & SD & $\mathrm{M}$ & SD \\
\hline & & 31.43 & 15.33 & 11.27 & 5.39 & 38.83 & 11.38 \\
\hline & & 29.50 & 12.72 & 10.24 & 5.85 & 43.70 & 8.64 \\
\hline & $t(p)$ & 0.65 & $(.52)$ & 0.82 & (.41) & -2.34 & $(.02)$ \\
\hline \multirow{5}{*}{ Age } & $18-30$ & 21.40 & 12.62 & 7.90 & 4.41 & 43.00 & 8.30 \\
\hline & $31-42$ & 32.15 & 12.14 & 10.59 & 6.03 & 42.10 & 10.21 \\
\hline & $43-54$ & 28.58 & 13.33 & 10.60 & 5.50 & 42.78 & 9.79 \\
\hline & $55+$ & 42.33 & 16.03 & 14.33 & 6.06 & 37.83 & 9.60 \\
\hline & $F(p)$ & 3.81 & $(.01)$ & 1.63 & $(.19)$ & 0.47 & $(.70)$ \\
\hline \multirow{5}{*}{ Working position } & Admin.staff & 24.67 & 11.92 & 8.07 & 3.24 & 40.27 & 9.18 \\
\hline & Scientific staff & 26.55 & 10.24 & 9.77 & 6.82 & 46.68 & 6.07 \\
\hline & Nursing staff & 31.07 & 12.27 & 9.48 & 4.85 & 44.85 & 8.59 \\
\hline & Other staff & 33.75 & 15.80 & 12.86 & 5.76 & 38.39 & 11.11 \\
\hline & $F(p)$ & 2.33 & $(.08)$ & 3.64 & $(.02)$ & 4.73 & $(.01)$ \\
\hline \multirow{5}{*}{ Educational level } & Elementary & 32.00 & 17.61 & 11.58 & 6.33 & 43.17 & 10.56 \\
\hline & High school & 32.03 & 14.35 & 11.51 & 5.36 & 39.26 & 11.33 \\
\hline & University & 28.24 & 11.74 & 9.32 & 5.41 & 44.39 & 7.37 \\
\hline & $\mathrm{MSc} / / \mathrm{PhD}$ & 27.13 & 11.38 & 10.63 & 7.63 & 44.37 & 8.73 \\
\hline & $F(p)$ & 0.73 & $(.54)$ & 1.14 & $(.34)$ & 2.12 & $(.10)$ \\
\hline \multirow{4}{*}{$\begin{array}{l}\text { Years of working } \\
\text { experience }\end{array}$} & $\leq 3$ & 24.89 & 13.18 & 10.05 & 5.54 & 40.32 & 12.34 \\
\hline & $4-9$ & 27.77 & 11.70 & 10.12 & 6.33 & 43.46 & 9.40 \\
\hline & $\geq 10$ & 32.96 & 13.88 & 10.93 & 5.53 & 42.33 & 8.97 \\
\hline & $F(p)$ & 3.17 & $(.04)$ & 0.26 & $(.77)$ & 0.57 & $(.57)$ \\
\hline \multirow{5}{*}{ Marital status } & Single & 23.86 & 11.02 & 8.43 & 4.20 & 43.43 & 11.24 \\
\hline & Married & 30.64 & 13.97 & 10.48 & 5.59 & 42.61 & 9.83 \\
\hline & Divorced & 34.18 & 11.34 & 13.73 & 7.16 & 38.18 & 6.34 \\
\hline & $F(p)$ & 2.10 & $(.13)$ & 2.77 & $(.07)$ & 1.12 & $(.33)$ \\
\hline & None & 23.75 & 10.09 & 8.50 & 3.89 & 44.15 & 9.71 \\
\hline \multirow{4}{*}{ Number of children } & One & 35.43 & 11.98 & 10.71 & 4.37 & 38.43 & 8.42 \\
\hline & Two & 30.67 & 12.60 & 11.45 & 6.82 & 44.48 & 8.87 \\
\hline & Three or more & 29.88 & 16.45 & 10.85 & 6.17 & 41.00 & 11.11 \\
\hline & $F(p)$ & 2.72 & $(.04)$ & 1.17 & $(.33)$ & 2.12 & $(.10)$ \\
\hline
\end{tabular}

Gender. Independent samples t-tests have found no significant effect of gender neither on the factor of emotional exhaustion, nor on the factor of Depersonalization with male participants scoring slightly higher than female participants in both cases. Nonetheless, the average score in Personal Accomplishment for men (38.83) has been proved significantly lower than that of women (43.7). Therefore, in this particular dimension, men show a higher degree of burnout than women.

Age. ANOVA analyses showed that the effect of age on the emotional exhaustion of the participants was overall significant. Through an adjustment according to Bonferonni concerning the number of comparisons, the only significant difference was detected between the age groups of "18 to 30" ( $M=21.4)$ and "55 and over" ( $M=42.3)$. The effect of age on the participants' depersonalization, as well as on the participants' feeling of personal accomplishment is not statistically significant.

Work position. The effect of the working position on the emotional exhaustion of the participants is not statistically significant, with 'other stuff' participants scoring slightly higher than 'nursing staff', 'scientific staff', and 'administrative staff'.

The effect of the working position on the participant's depersonalization, though, is deemed statistically significant. Through an adjustment according to Bonferonni for the number of comparisons, the only significant difference was detected between administrative $(M=8.06)$ and other staff $(M=12.86)$. 
The effect of the working position on the sub-scale of the participants' feeling of personal accomplishment is also found statistically significant. Through an adjustment according to Bonferonni, statistically significant differences were found between scientific and other staff and between nursing and other staff. Namely, the participants falling under the scientific $(M=46.68)$ and nursing staff $(\mathrm{M}=44.85)$, show less degree of burnout regarding this sub-scale, compared to the participants falling under the other staff $(M=38.38)$.

Education. The effect of the educational level is not statistically significant on emotional exhaustion, nor on depersonalization, nor the "feeling of personal accomplishment". Similarly, the effect of marital status is not statistically significant in any of the subscales of burnout.

Work experience. Working experience does affect significantly emotional exhaustion with more years of experience producing more exhaustion. However, working experience does not seem to affect significantly depersonalization nor the "feeling of personal accomplishment".

Children. The effect of the number of the participants' children on emotional exhaustion is also statistically significant. Post-hoc analyses according to Bonferonni showed that, a statistically significant difference was observed between the category of participants who do not have children and the group of employees who have one child. Namely, participants who do not have children (M $=23.75$ ) show lower degree of emotional exhaustion compared to employees who have one child $(\mathrm{M}=35.42)$. Participants with two children or with three or more children are not significantly differentiated between them or from the other groups. Finally, the effect of the number of the children is not statistically significant on the participants' depersonalization, nor on the "feeling of personal accomplishment".

On an additional set of analyses based on the number of people experiencing or not burnout syndrome symptoms, we investigated its relation to the participants' demographic characteristics (see, Table 4).

Table 4. Demographics effect on Burnout syndrome

\begin{tabular}{|c|c|c|c|c|c|c|c|}
\hline & \multicolumn{4}{|c|}{ Burnout Syndrome } & \multirow[b]{3}{*}{$x^{2}$} & \multirow[b]{3}{*}{$p$} \\
\hline & & \multicolumn{2}{|c|}{ Yes } & \multicolumn{2}{|c|}{ No } & & \\
\hline & & $\mathrm{N}$ & $\%$ & $\mathrm{~N}$ & $\%$ & & \\
\hline \multirow{2}{*}{ Sex } & Male & 9 & $30.0 \%$ & 21 & $70.0 \%$ & \multirow{2}{*}{10.95} & \multirow{2}{*}{.001} \\
\hline & Female & 4 & $5.7 \%$ & 66 & $94.3 \%$ & & \\
\hline \multirow{4}{*}{ Age } & $18-30$ & 0 & $0.0 \%$ & 10 & $100.0 \%$ & \multirow{4}{*}{4.21} & \multirow{4}{*}{.240} \\
\hline & $31-42$ & 4 & $10.3 \%$ & 35 & $89.7 \%$ & & \\
\hline & $43-54$ & 7 & $15.6 \%$ & 38 & $84.4 \%$ & & \\
\hline & 55 and above & 2 & $33.3 \%$ & 4 & $66.7 \%$ & & \\
\hline \multirow{4}{*}{ Working position } & Administrative staff & 1 & $6.7 \%$ & 14 & 93.3 & \multirow{4}{*}{8.49} & \multirow{4}{*}{.037} \\
\hline & Scientific staff & 0 & $0.0 \%$ & 22 & $100.0 \%$ & & \\
\hline & Nursing staff & 3 & $11.1 \%$ & 24 & $88.9 \%$ & & \\
\hline & Other staff & 9 & $25.0 \%$ & 27 & $75.0 \%$ & & \\
\hline \multirow{4}{*}{ Educational level } & Elementary school & 3 & $25.0 \%$ & 9 & 75.0 & \multirow{4}{*}{10.27} & \multirow{4}{*}{.016} \\
\hline & High school & 9 & $23.1 \%$ & 30 & $76.9 \%$ & & \\
\hline & University/Technological Educational Institute & 1 & $2.4 \%$ & 40 & $97.6 \%$ & & \\
\hline & $\mathrm{MSc} / / \mathrm{PhD}$ & 0 & $0.0 \%$ & 8 & $100.0 \%$ & & \\
\hline \multirow{3}{*}{$\begin{array}{l}\text { Years of working } \\
\text { experience }\end{array}$} & Up to three & 2 & $10.5 \%$ & 17 & $89.5 \%$ & \multirow{3}{*}{0.24} & \multirow{3}{*}{.888} \\
\hline & From four to nine & 4 & $15.4 \%$ & 22 & $84.6 \%$ & & \\
\hline & Ten or more & 7 & $12.7 \%$ & 48 & $87.3 \%$ & & \\
\hline \multirow{4}{*}{ Marital status } & Single & 2 & $14.3 \%$ & 12 & $85.7 \%$ & \multirow{4}{*}{0.35} & \multirow{4}{*}{.840} \\
\hline & Married & 9 & $12.0 \%$ & 66 & $88.0 \%$ & & \\
\hline & Divorced & 2 & $18.2 \%$ & 9 & $81.8 \%$ & & \\
\hline & Widower & 0 & $0.0 \%$ & 0 & $0.0 \%$ & & \\
\hline & None & 2 & $10.0 \%$ & 18 & 90.0 & \multirow{4}{*}{1.53} & \multirow{4}{*}{.676} \\
\hline \multirow{3}{*}{ Number of children } & One & 3 & $14.3 \%$ & 18 & $85.7 \%$ & & \\
\hline & Two & 3 & $9.1 \%$ & 30 & $90.9 \%$ & & \\
\hline & Three or more & 5 & $19.2 \%$ & 21 & $80.8 \%$ & & \\
\hline
\end{tabular}


By using the Pearson's $x^{2}$ test, it is initially shown that the percentage of men who suffer burnout syndrome is higher $\left(x^{2}=10.96, p=.001\right)$ than of women ( $30 \%$ and $5.7 \%$, respectively). Besides, the participants falling under the working category of other staff show significantly higher burnout syndrome percentage than the rest individuals as they reach $25 \%\left(x^{2}=8.49, p=.037\right)$. Though, lower-educated employees namely Elementary school and High school graduates show quite high percentages of burnout syndrome as these are equal to $25 \%$ and $23.1 \%$ respectively $\left(x^{2}=10.27, p\right.$ $=.016)$.

\subsection{The Effect of Demographic Factors on Depressive Symptomatology}

Table 5 summarizes the average scores (and standard deviations) of depressive symptomatology according to the demographic characteristics of the participants, as well as the inductive analyses results on the significance of their effect.

Table 5. Demographics effect on depressive symptomatology score

\begin{tabular}{|c|c|c|c|c|}
\hline \multirow{4}{*}{ Sex } & \multirow{4}{*}{$\begin{array}{l}\text { Male } \\
\text { Female }\end{array}$} & \multicolumn{3}{|c|}{ Depressive Symptomatoloy } \\
\hline & & $\mathrm{M}$ & SD & $p$ \\
\hline & & 12.00 & 9.05 & \multirow{2}{*}{1.41 .162} \\
\hline & & 9.31 & 8.60 & \\
\hline \multirow{5}{*}{ Age } & & & & $F \quad p$ \\
\hline & $18-30$ & 8.90 & 7.20 & \multirow{4}{*}{0.58 .630} \\
\hline & $31-42$ & 9.23 & 7.76 & \\
\hline & $43-54$ & 10.69 & 9.96 & \\
\hline & $55+$ & 13.67 & 8.69 & \\
\hline \multirow{4}{*}{ Working position } & Administrative staff & 8.20 & 6.74 & \multirow{4}{*}{4.41 .006} \\
\hline & Scientific staff & 6.95 & 5.79 & \\
\hline & Nursing staff & 8.44 & 6.25 & \\
\hline & Other staff & 14.11 & 11.16 & \\
\hline \multirow{4}{*}{ Educational level } & Eleentary school & 11.08 & 8.40 & \multirow{4}{*}{2.39 .073} \\
\hline & High school & 12.64 & 10.99 & \\
\hline & University/Technological Educational Institute & 8.20 & 6.26 & \\
\hline & $\mathrm{MSc} / / \mathrm{PhD}$ & 6.25 & 4.95 & \\
\hline \multirow{3}{*}{$\begin{array}{l}\text { Years of working } \\
\text { experience }\end{array}$} & Up to three & 9.26 & 6.76 & \multirow{3}{*}{1.12 .330} \\
\hline & From four to nine & 8.31 & 6.03 & \\
\hline & Ten or more & 11.27 & 10.31 & \\
\hline \multirow{3}{*}{ Marital status } & Single & 7.64 & 5.11 & \multirow{3}{*}{0.66 .522} \\
\hline & Married & 10.47 & 9.47 & \\
\hline & Divorced & 10.91 & 7.48 & \\
\hline \multirow{4}{*}{ Number of children } & None & 6.65 & 5.10 & \multirow{4}{*}{1.93 .131} \\
\hline & One & 11.05 & 5.95 & \\
\hline & Two & 9.64 & 8.39 & \\
\hline & Three or more & 12.65 & 12.25 & \\
\hline
\end{tabular}

The effect of gender and age on depressive symptomatology was not statistically significant. The effect of the working position on the participants' depressive symptomatology was overall significant $(F=4.41, p=.06)$. Through an adjustment according to Bonferonni for the number of comparisons, the only significant difference $(p=.013)$ was found between scientific $(M=6.95$, SD $=5.78)$ and other staff $(M=14.11, S D=11.15)$. It is shown that the participants who fall under the scientific staff have less depressive symptomatology compared to employees who fall under other staff. Finally, the effect of the educational level, professional experience, marital status and the number of children of the participants on depressive symptoms are not deemed significant.

\subsection{The Relationship between Burnout and Depressive Symptomatology}

Table 6 presents the Pearson's correlations conducted in order to investigate the relationships 
between depressive symptomatology and the factors of Burnout. The results indicate that there is a positive and statistically significant correlation between Emotional exhaustion and Depressive Symptomatology and between Depersonalization and Depressive Symptomatology. In other words, high levels of Emotional Exhaustion and Depersonalization are associated with elevated levels of Depressive Symptomatology. On the contrary, Personal accomplishment and Depressive Symptomatology are significantly but negatively correlated, since higher levels of depressive symptomatology are associated with lower levels of personal accomplishment and vice-versa.

Table 6. Correlation coefficients (Pearson's $r$ ) between burnout factors and depressive symptomatology scores

\begin{tabular}{lccc} 
& Depressive sympt. & Emotional exhaustion & Depersona-lization \\
\hline Emotional exhaustion & $.616^{\pi \pi}$ & & \\
Depersonalization & $.400^{\pi \pi}$ & $.479^{\pi \pi}$ & $-.562^{\pi \pi}$ \\
Personal accomplishment & $-.489^{\pi \pi}$ & $-.456^{\pi \pi}$ & \\
\hline
\end{tabular}

${ }^{* *}$. Correlation is significant at the .001 level (2-tailed).

Finally, an independent samples t-test revealed that the individuals that experience Burnout Syndrome show significantly higher levels of Depressive Symptomatology $(M=20.46, S D=8.31)$ than the employees who do not suffer from Burnout Syndrome $(M=8.57, S D=7.78)(t=5.09, p<$ 0.001).

\section{Discussion}

Regarding the three dimensions of burnout, two out of three workers show medium to high emotional exhaustion, four out of five workers show medium to high depersonalization and two out of five show medium to high burnout, as for the dimension of "feeling of personal accomplishment". These percentages indicate significant burnout of the employees in the Institutions of Chronic Diseases, such as other researches in Greek and foreign healthcare institutions, although the percentages attributed to each of the three dimensions of burnout differ importantly between the researches (e.g. Ackerley et al. 1988; Queiros et al. 2013; Noula et al. 2010). Apart from the different methodological approach of the researchers, this can be interpreted as a dependence of burnout's syndrome mostly on factors of working framework and less on personal and other nonworking factors.

Regarding the depressive symptomatology, as measured by the specific psychometric tool used, two out of three workers show depressive symptoms, ranging from very light to heavy. Compared to this percentage, in some studies have been found lower rates, in some similar or higher, depending on the specialisation of the staff and the evaluation manner of the depressive symptomatology (e.g. Siebert 2004; Yoon and Kim 2013; Chang et al. 2010). These differences can be attributed both to the different conditions existing in different socio-cultural environments of the research's implementation and to the research's chosen methodology.

Concerning the influence of demographic variables, statistically significant results were recorded in all three dimensions of burnout. Men employees show a higher degree of burnout, compared to the women, in the aspect of "feeling of personal accomplishment". The international literature indicates that the role gender has not been adequately clarified (Pappa et al. 2007; Vredenburgh et al. 1999). However, this finding may be owed to the traditional notions of gender, still existing to a certain extent in Greece, which gives the role of the "breadwinner" to the man, with any pressure this position may entail.

The employees from 55 and older experience emotional exhaustion to a greater degree than the workers aged 18-30. The same was found in the research of Stanetić and Tesanović (2013), which was conducted by medical staff, although the opposite has also been found in other researches (Vredenburgh et al. 1999; Hamama 2012). The specific finding of the present study could be interpreted by the prolonged age, by which their families are financially supporting many young people in Greece. Therefore, the severe conditions at work are not encountered as a threat 
to them.

The employees in the category of administrative staff showed a lesser extent of depersonalization than the employees in the group of other employees. The role of job characteristics has been found to be critical for the development of the burnout syndrome (Demerouti et al. 2001; Lee and Ashforth 1993). Deremouti and her colleagues (2001) suggested that human service professionals may feel exhausted by the emotionally demanding contacts with their patients and treat them in a depersonalised way. However, in other occupations, manifestations may differ, since there are no recipients of one's service who can be dealt with in a depersonalised manner. Similarly, since the administrative staff of the Institutions for Chronic Diseases has little or no interaction with the patients, it is likely to be less depersonalised.

Finally, the results of the research showed that employees who do not have a child show a lesser degree of emotional exhaustion compared to employees who have a child. Similarly, in a study conducted among nurses within a hospital in Cyprus (Mpaltzi et al. 2012) it was found that most children of employees were associated with greater emotional exhaustion and burnout in general. Specifically, in Greece, this result seems to be related to the current economic situation. Through the years of economic crisis, the supportive structures for the family are limited. Furthermore, the presence of children constitutes an additional factor which creates pressure to workers, because of the increased financial liabilities.

Regarding the influence of the demographic variables on depressive symptomatology, the employees in the scientific staff show less depressive symptomatology compared to employees in other auxiliary staff. This finding agrees with the results of other studies (Siebert 2004; Kaklamanou et al. 2014), indicating that the working position associated with a particular educational level, can exert a protective effect.

According to the results, employees who experience burnout syndrome show statistically significant higher levels of depressive symptomatology than the employees who do not suffer from the syndrome. The relationship between these two health factors have been verified in several studies (e.g., Brenninkmeyer et al. 2001; lakovides et al. 2003; Plieger et al. 2015). Furthermore, Schonfeld and Bianchi (2016) conclude that the state of burnout is likely to be a form of depression. The controversial relations between these two factors and the possible overlap of them as they develop over time need to be unravelled, probably focusing on further longitudinal studies.

The specific research used a cross-sectional design and had a sample of employees from four Institutions of Chronic Diseases in semi-rural areas. Therefore, it is not possible to generalise the findings and they not necessarily relate to the employees within Institutions of Chronic Diseases in the major urban centres. Furthermore, the use of self-report measures may have limited the accuracy of the responses and biased the results. Finally, a small percentage of all the possible factors or mediators have been studied. Therefore, other variables that were not simultaneously considered may contribute or interfere and affect the presented results.

\section{Conclusion}

The existence of the problem and the general vulnerability in burnout and depressive symptomatology of employees within Institutions of Chronic Diseases was demonstrated through this research. Therefore, based on these results, it is necessary to make some interventions, on a preventive level as well as to deal with burnout problems and depression of employees. The results of the effect of demographic variables on the dependent variables do not indicate the existence of a vulnerable employee profile. Therefore, any intervention may be adopted, may concern all the staff. Consequently, it is necessary to design preventive intervention of timely diagnosis and treatment of problems related to the mental well-being of the workers within Institutions of Chronic Diseases, and in future researches evaluation of the effectiveness of these interventions. Additionally, employees' increased burnout should call the attention for possible depressive symptomatology and therefore, a direct psychological assessment would be suggested. Integrated interventions, aimed at health and effectiveness of the employees would benefit indirectly, beyond the staff, the structures and mainly the cared population, which should then be studied and evaluated, to have a particular scientific aspect and comprehensive understanding of the issue. 


\section{References}

Abbas, M.A.F., Abu Zaid, L.Z., Hussaein, M., Bakheet, K.H., \& Alhamdan, N.A. (2013). Anxiety and depression among nursing staff at King Fahad Medical City, Kingdom of Saudi Arabia. In: The 2013 WEI International Academic Conference Proceedings. Istanbul, Turkey: The West East Institute. [Online] Available: https://www.westeastinstitute.com/wp-content/uploads/2013/07/Lamiaa-Z.pdf. (May 10, 2017).

Ackerley, G. D., Burnell, J., Holder, D. C., \& Kurdek, L. A. (1988). Burnout among licensed psychologists. Professional Psychology: Research and Practice, 19(6), 624-631.

Ahola, K., Hakanen, J., Perhoniemi, R. \& Mutanen, P. (2014). Relationship between burnout and depressive symptoms: a study using the person-centred approach. Burnout Research, 1, 29-37. doi: 10.1016/j.burn.2014.03.003

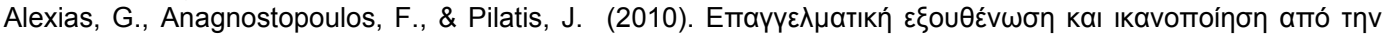

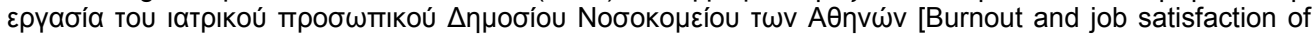

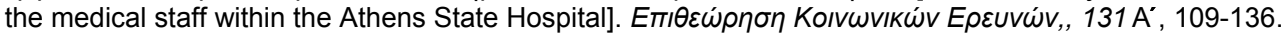

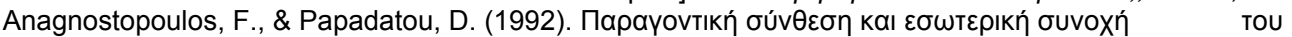

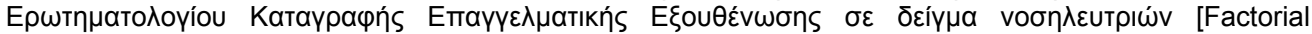
composition and internal consistency of the Questionnaire of Burnout's Recording within a sample of

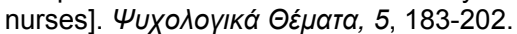

Bakker, A.B., Schaufeli, W.B., Demerouti, E., Janssen, P.P.M., Hulst, R.V.D., \& Brouwer, J. (2000). Using equity theory to examine the difference between burnout and depression. Anxiety, Stress and Coping, 13, 247268.

Beck, A.T., Ward, C.H., Mendelson, M., Mock, J., \& Erbaugh, J. K. (1961). An inventory for measuring depression. Archives of general psychiatry, 4(6), 561-571.

Bianchi, R., Schonfield, I.S., \& Laurent, E. (2015). Burnout-depression overlap: a review. Clinical Psychology Review, 36, 28-41. doi: 10.1016/j.cpr.2015.01.004.

Brenninkmeyer, V., Van Yperen, N.W., \& Buunk, B. (2001). Burnout and depression are not identical twins: is decline of superiority a distinguishing feature? Personality and Individual Differences, 30, 873-880.

Chang, Y., Wang, P., Li, H., \& Liu, Y. (2010). Relations among depression, self-efficacy and optimism in a sample of nurses in Taiwan. Journal of Nursing Management, 19, 769-776. doi: 10.1111/j.13652834.2010.01180.x

Demerouti, E., Bakker, A.B., Nachreiner, F., \& Schaufeli, W.B. (2001). The job demands-resources model of burnout. Journal of Applied Psychology, 86(3), 499-512.

Fong, T.C.T., Ho, R.T.H., Au-Yeung, F.S.W., Sing, C.Y., Law, K.Y., Lee, L.F. \& Ng, S.M. (2016). The relationships of change in work climate with changes in burnout and depression: a 2-year longitudinal study of Chinese mental health care workers. Psychology, Health and Medicine, 21(4), 401-412. doi: http://dx.doi.org/10.1080/13548506.2015.1080849

Gao, Y., Pan, B., Sun, W., Wu, H., Wang, J., \& Wang, L. (2011). Depressive symptoms among Chinese nurses: prevalence and the associated factors. Journal of Advanced Nursing, 68(5), 1166-1175. doi: 10.1111/j.1365-2648.2011.05832.x

Hamama, L. (2012). Burnout in social workers treating children as related to demographic characteristics, work environment, and social support. Social Work Research, 36(2), 113-125. doi: 10.1093/swr/svs003.

lakovides, A., Fountoulakis, K.N., Kaprinis, S. \& Kaprinis, G. (2003). The relationship between job stress, burnout and clinical depression. Journal of Affective Disorders, 75, 209-221.

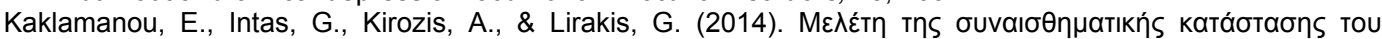

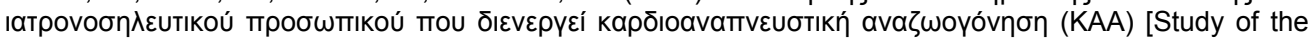

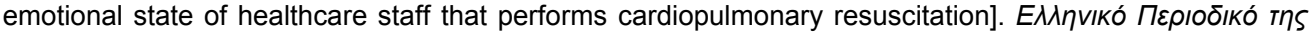

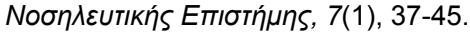

Koinis, A., Tziaferi, S., \& Saridi, M. (2014). Mental health problems in health professionals. Interscientific Health Care, 6(1), 8-17.

Kyloudis, P., Rekliti, M., Kyriazis, I., Wozniak, G., Kotrotsiou, E., \& Roupa, Z. (2010). Probing stress and depression in mental health professionals at the general hospital in Greece. European Psychiatry, 25: 731. doi: $10.1016 /$ S0924-9338(10)70725-2.

Lee, R.T. \& Ashforth, B.E. (1993). A longitudinal study of burnout among supervisors and managers: comparisons between the Leiter and Maslach (1988) and Golembiewski et al. (1986) Models. Organizational Behaviour and Human Decision Processes, 54, 369-398.

Leiter, M.P., \& Durup, J. (1994). The discriminant validity of burnout and depression: a confirmatory factor analytic study. Anxiety, Stress and Coping, 7, 357-373.

Levine, R.E., \& Bryant, S.G. (2000). The depressed physician: a different kind of impairment. Hospital Physician, 36, 67-73.

Maslach, C. \& Jackson, S.E. (1981). MBI- Human Services Survey- MBI-HSS: Copyright $\odot$ 1981. All rights 
reserved in all media. Published by Mind Garden, Inc., www.mindgarden.com

Maslach, C., Jackson S.E., \& Leiter, M. (1997). The Maslach Burnout Inventory Manual. Chapter (PDF Available). In C. P. Zalaquett, R. J. Wood, (Eds), Evaluating Stress: A Book of Resources (pp. 191-218). Lanhan, MD: The Scarecrow Press. [Online] Available: https://www.researchgate.net/profile/Christina_Maslach/publication/277816643_The_Maslach_Burnout_In ventory Manual/links/5574dbd708aeb6d8c019̄46d7.pdf. (May 18, 2017).

Mpaltzi, E., Hari-Papaioannou, F., Polikandrioti, M., Gourni, M., \& Charalampous, G. (2012). Exploration of burnout syndrome in general hospitals in Larnaka of Cyprus. Rostrum of Asclepius, 11 (4), 531-548.

Mutkins, E., Brown, R.F., \& Thorsteinsson, E.B. (2011). Stress, depression, workplace and social supports and burnout in intellectual disability support staff. Journal of Intellectual Disability Research, 55(5), 500-510. doi: 10.1111/j.1365-2788.2011.01406.x.

Noula, M., Mouhaki, S., Argiropoulou, D., Ifanti, E., Kiriakidou, V., Tsiriga S., \& Gesouli, E. (2010). H

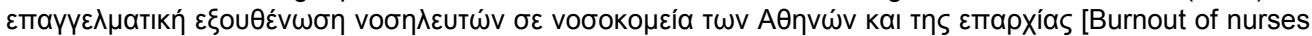
within hospitals in Athens and province]. Interscientific Health Care, 2(2), 99-103.

Official Gazette of the Hellenic Republic (2009). Presidential Decree No 154/2009, Gov. no. sheet No 196/A/0110-2009. [Online] Available: http://www.et.gr/idocs$\mathrm{nph} /$ search/pdfViewerForm.html?args=5C7QrtC22wEilCErm5tbxndtvSoCIrL8H69BYATHe5V5MXD0LzQT LWPU9yLzB8V68knBzLCmTXKaO6fpVZ6Lx3UnKI3nP8NxdnJ5r9cmWyJWeIDvWS_18kAEhATUkJb0x1L IdQ163nV9K--td6SludEGKQ4_K2tn5Cg4I9r_GC5_uzPU2RpBn5hUMu5EbpzH..(August 4, 2017).

Parekh, R. (2017). What is depression? American Psychiatric Association. [Online] Available: https://www.psychiatry.org/patients-families/depression/what-is-depression. (July 4, 2017).

Pappa, E.A., Anagnostopoulos, F., \& Niakas, D. (2007). Burnout of medical and nursing staff and its impact on

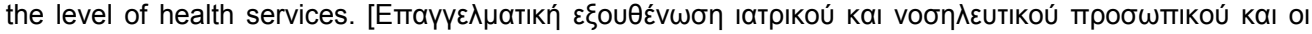

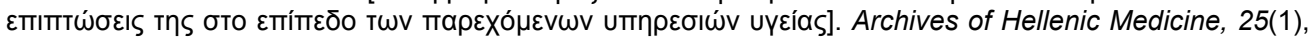
94-101.

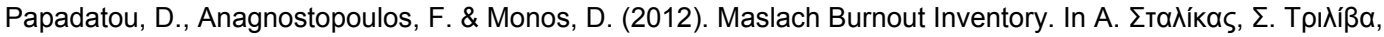

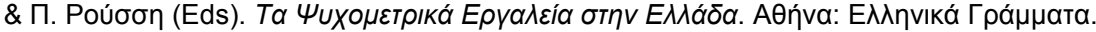

Plieger, T., Melchers, M., Montag, C., Meermann, R. \& Reuter, M. (2015). Life stress as potential risk factor for depression and burnout. Burnout Research, 2, 19-24. doi: 10.1016/j.burn.2015.03.001.

Queiros, C., Carlotto, M.S., Kaiseler, M., Dias, S., \& Pereira, M. (2013). Predictors of burnout among nurses: an interactionist approach. Psicothema, 25(3), 330-335. doi: 10.7334/psicothema2012.246.

Rios, K.A., Barbosa, D.A., \& Belasco, A.G.S. (2010). Evaluation of quality of life and depression in nursing technicians and nursing assistants. Revista Latino-Americana de Enfermagem, 18(3), 413-420. [Online] Available: http://www.scielo.br/pdf/rlae/v18n3/17.pdf. (May 14, 2017).

Schaufeli, W.B., \& Buunk, B.P. (2003). Burnout: an overview of 25 years of research and theorizing. In M.J. Schabracq, J.A.M. Winnubst, \& C.L. Cooper (Eds.), The Handbook of work and health psychology (pp. 383-425). Chichester England: Wiley.

Schonfeld, I.S. \& Bianchi, R. (2016). Burnout and depression: two entities or one? Journal of Clinical Psychology, 72(1), 22-37. doi: 10.1002/jclp.22229.

Siebert, D.C. (2004). Depression in North Carolina social workers: implications for practice and research. Social Work Research, 28, 30-40.

Stanetić, K., \& Teŝanović, G. (2013). Influence of age and length of service on the level of stress and burnout syndrome. Medicinski Pregled, 66(3-4), 153-162.

Toker, S. \& Biron, M. (2012). Job burnout and depression: unraveling their temporal relationship and considering the role of physical activity. Journal of Applied Psychology, 97(3), 699-710. doi: 10.1037/a0026914.

Tragea, P., Damigos, D., Mavreas, V., \& Gouva, M. (2012). Burn out among Greek physical therapists. Interscientific Health Care, 4(2), 77-82.

Tselebis, A., Gournas, G., Tzitzanidou, G., Panagiotou, A., \& Ilias, I. (2006). Anxiety and depression in Greek nursing and medical personnel. Psychological Reports, 99(1), 93-96. doi: 10.2466/pr0.99.1.93-96.

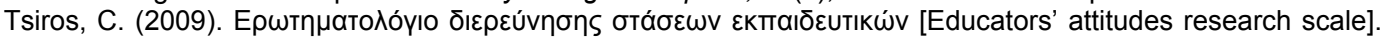
[Online]

https://www.google.gr/url?sa=t\&rct=j\&q=\&esrc=s\&source=web\&cd=1\&ved=0ahUKEwi7-

pvP9tDSAhWGXRQKHV-

aBRoQFggaMAA\&url=http\%3A\%2F\%2Fkatsiras.mysch.gr\%2Findex.php\%3Foption\%3Dcom_docman\%2 6task\%3Ddoc_download\%26gid\%3D24\%26Itemid\%3D16\&usg=AFQjCNFJDvSQC5ItiEUx1CkYXnD_dJ5 O_Q. (June 19, 2017).

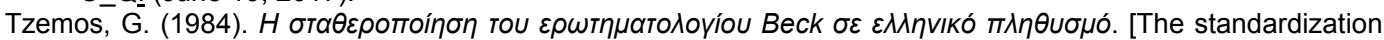

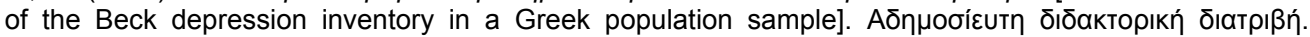

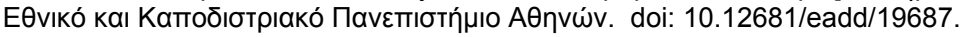


Vredenburgh, L.D., Carlozzi, A.F., \& Stein, L.B. (1999). Burnout in counseling psychologists: type of practice setting and pertinent demographics. Counselling Psychology Quarterly, 12(3), 293-302.

Yoon, S.L., \& Kim, J. (2013). Job-relates stress, emotional labor, and depressive symptoms among Korean nurses. Journal of Nursing Scholarship, 45 (2), 169-176. doi: 10.1111/jnu.12018. 